\title{
GAMMA-RAY BURST JET DYNAMICS
}

\author{
J. Granot ${ }^{1}$
}

\begin{abstract}
This is a brief review of some recent progress in our understanding of GRB jet dynamics, during the early acceleration phase and the later afterglow phase. In the acceleration phase I focus on the possible role of impulsive magnetic acceleration, and its ability to convert most of the initial magnetic energy into kinetic energy and naturally produce efficient internal shocks at mild magnetizations. For the afterglow phase I outline new generalized yet simple analytic models that finally agree with numerical simulations, and present recent simulation results for a jet propagating into a stratified external medium.
\end{abstract}

\section{Introduction}

GRB outflows are expected to be collimated into narrow bipolar jets, in analogy to other astrophysical relativistic outflow sources, such as active galactic nuclei (AGN) and micro-quasars (e.g., Rhoads 1997). However, unlike some of these other sources GRB outflows are almost always unresolved, point sources, so there is only indirect evidence for jets in GRBs. Collimation into narrow jets can alleviate the "energy crisis" that arises from their very large energy outputs in $\gamma$-rays assuming isotropic emission, $E_{\gamma \text {,iso }}$ (the current record being $E_{\gamma \text {,iso }} \approx 4.9 M_{\odot} c^{2}$ for GRB 080916C; Abdo et al. 2009). If most of the $\gamma$-rays are emitted within a small fraction, $f_{b} \ll 1$, of the total solid angle (where $f_{b} \approx \theta_{0}^{2} / 2$ for conical uniform narrow bipolar jets of initial half-opening angle $\theta_{0}$ ), then the true energy output in $\gamma$-rays, $E_{\gamma}$, is much smaller than its isotropic equivalent value, $E_{\gamma}=f_{b} E_{\gamma \text {,iso }}$.

The angular structure of GRB outflows is important for inferring their true energy output and event rate, as well as properties of their central engine. Moreover, the jet structure and dynamics are crucial for correctly modeling and interpreting observations, and inferring from them important physical parameters such as the external density profile and the microphysical parameters of relativistic collisionless shocks. Nevertheless, the jet angular structure is still not very well constrained

1 Department of Natural Sciences, The Open University of Israel, 1 University Road, PO Box 808, Raanana 43537, Israel 
observationally, despite various efforts (e.g., Granot 2005, 2007). Here I focus on the dynamics of a double-sided jet that is initially uniform with sharp edges and a half-opening angle $\theta_{0} \ll 1$, which is the most widely studied jet angular structure.

The dynamics of GRB jets can be divided into several different parts or stages:

- Launching: the launching of the jet is likely magnetic, possibly due to the Blandford-Znajek mechanism operating in a newly formed, rapidly accreting stellar mass black hole, or an MHD pulsar-type wind for a millisecond magnetar central engine; neutrino - anti-neutrino annihilation may also play an important role. This first stage is still not very well understood.

- Acceleration: the two main candidates for the dominant acceleration mechanism are thermal acceleration (by the radiation pressure in an optically thick electron-positron, photon and baryon plasma - the fireball model) and magnetic acceleration (which is discussed in Sect. 2).

- Propagation inside the progenitor star (for GRBs of the long-soft class).

- Collimation: the jet collimation can be assisted by the interaction with the external medium, and in particular with the progenitor star for long GRBs, by the accretion disk wind, and by magnetic hoop stress.

- Coasting phase, which ends at the deceleration radius $R_{\mathrm{dec}}$ (expected for thermal acceleration, but does not always exist for magnetic acceleration).

- Relativistic self-similar: at $R>R_{\text {dec }}$ most of the energy is in the shocked external medium, the outflow composition and radial profile are essentially forgotten, but the angular profile persists. Locally, however, the flow approaches the Blandford \& McKee (1976) spherical self-similar solution.

- Sideways expansion: once $\Gamma$ drops below $1 / \theta_{0}$, at radii $R>R_{\mathrm{j}}$, significant jet lateral expansion is possible, but it is unclear to what extent it occurs in practice (this is discussed in detail in Sect. 3).

- Newtonian self-similar: eventually the flow becomes Newtonian and spherical, approaching the Sedov-Taylor self-similar solution.

Here the focus is on recent progress in our understanding of impulsive magnetic acceleration (in Sect. 2), and of the jet dynamics during the afterglow stage (in Sect. 3).

\section{Magnetic acceleration: The role of strong time dependence}

The two main competing acceleration mechanisms for GRB outflows are thermal and magnetic acceleration. Moreover, magnetic acceleration likely plays a key role also in other relativistic jet sources, such as AGN or micro-quasars. It was realized early on that the collimation and acceleration of initially very hot and high-pressure material near the source to highly super-sonic speeds (e.g. the 
"twin exhaust" model for AGN jets; Blandford \& Rees 1974) faces difficulties under realistic astrophysical conditions (it is subject to various instabilities; Smith et al. 1981). Hence, magnetic fields play an important role in many models for the launching, collimation and acceleration of relativistic jets. Most such models assume a steady flow, both as this may adequately describe outflows that vary slowly enough with time, and since it significantly simplifies the relevant dynamical equations and allows analytic self-similar solutions (e.g. Begelman \& Li 1992; Vlahakis \& Königl 2003). Strong magnetic fields near the source may also help avoid excessive mass loading and thus enable the jets to reach relativistic speeds.

A highly magnetized steady spherical flow accelerates only up to an asymptotic Lorentz factor $\Gamma_{\infty} \sim \sigma_{0}^{1 / 3}$ and magnetization $\sigma_{\infty} \sim \sigma_{0}^{2 / 3}$ (Goldreich \& Julian 1970) where $\sigma_{0} \gg 1$ is the initial value of the magnetization parameter $\sigma$ (the ratio of electromagnetic to matter energy fluxes or enthalpy densities), i.e. most of the energy remains in electromagnetic form (a Poynting flux dominated flow). Collimation by an external pressure leading to an asymptotic jet half-opening angle $\theta_{\mathrm{j}}$ can increase $\Gamma_{\infty}$ and decrease $\sigma_{\infty}$ by up to a factor of $\sim \theta_{\mathrm{j}}^{-2 / 3}$, since lateral causal contact in the jet is maintained if $\theta_{\mathrm{j}}$ does not exceed the Mach angle $\left(\theta_{\mathrm{j}} \lesssim \theta_{\mathrm{M}} \sim \sigma^{1 / 2} / \Gamma\right.$, where energy conservation implies $\sigma \Gamma \sim \sigma_{0}$ as long as the flow remains highly magnetized, $\sigma \gg 1$ ). However, even under the most favorable conditions the asymptotic magnetization is $\sigma_{\infty} \geq 1$, which does not allow efficient energy dissipation in internal shocks within the outflow (Lyubarsky 2009, 2010a; Komissarov et al. 2009). A sudden drop in the external pressure, as may occur e.g. when a GRB jet exits its progenitor star, can result in a sudden additional acceleration (Komissarov et al. 2010; Tchekhovskoy et al. 2010) that can lead to $\Gamma_{\infty} \theta_{\mathrm{j}} \gg 1$ as inferred in GRBs, but still with $\sigma_{\infty} \geq 1$.

These limitations of the "standard" steady, axi-symmetric and non-dissipative (or ideal MHD) magnetic acceleration have, on the one hand, led to the suggestion that the jets might remain Poynting flux dominated at large distances from the source and the observed emission is the result of magnetic reconnection events rather than internal shocks (Blandford 2002; Lyutikov 2006). On the other hand, other models suggested increasing the acceleration efficiency by relaxing one of the standard assumptions, such as axi-symmetry - leading to non-axi-symmetric instabilities that randomize the magnetic field orientation (Heinz \& Begelman 2000). A highly tangled magnetic field effectively behaves like a relativistic fluid (with an adiabatic index of $4 / 3$ ) and leads to efficient acceleration, similar to thermal acceleration of relativistic outflows. Moreover, both the kink instability mentioned above (Drenkhahn \& Spruit 2002), as well as other instabilities (such as the Kruskal-Schwarzschild instability in a striped wind; Lyubarsky 2010b) can lead to magnetic reconnection, i.e. gradual magnetic dissipation, which in turn enhances the acceleration due to the conversion of magnetic to thermal energy, where the thermal pressure efficiently accelerates the outflow.

A natural alternative is replacing the usual steady-state assumption by strong time-dependence. This impulsive regime was sparsely studied, and mainly in the non-relativistic case (Contopoulos 1995). Recently, a new impulsive magnetic 
acceleration mechanism was found that operates in the relativistic case (Granot et al. 2011), which can be much more efficient than magnetic acceleration in steady flows, and can lead to low magnetizations, $\sigma \ll 1$, thus enabling efficient dissipation in internal shocks. This qualitatively different behavior of impulsive outflows can be very relevant for GRBs, as well as for other relativistic jet sources such as tidal disruptions or flares in AGN or micro-quasars, or even giant flares in soft gamma repeaters (SGRs, thought to be magnetars - highly magnetized neutron stars). It also triggered renewed interest in this topic (e.g. Granot 2012a,b; Komissarov 2012; Levinson 2010; Lyutikov 2011).
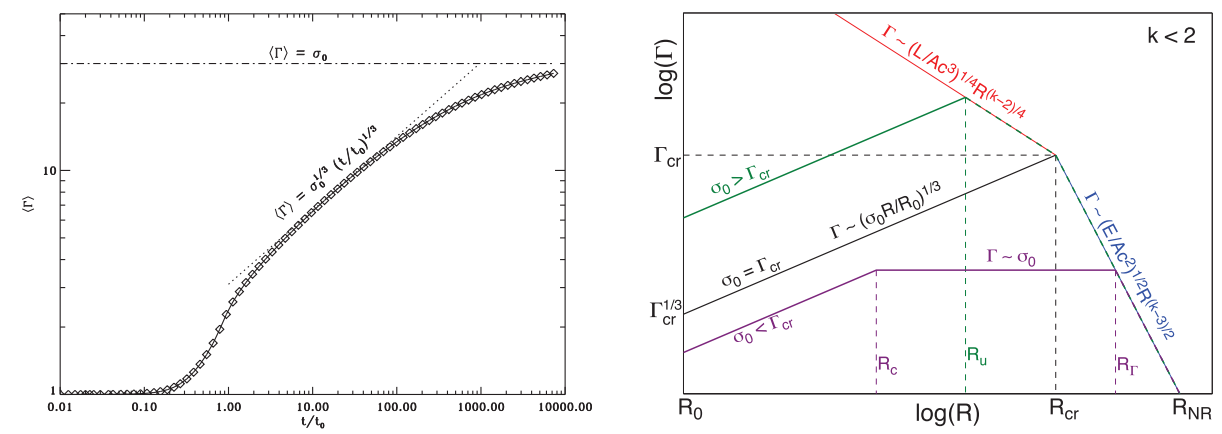

Fig. 1. Left: test case for impulsive magnetic acceleration: the energy-weighted mean Lorentz factor $\langle\Gamma\rangle$ of a finite cold shell of plasma initially uniform (with with $l_{0}$, rest mass density $\rho_{0}$ and magnetic field $\left.B_{0}\right)$, highly magnetized $\left(\sigma_{0}=B_{0}^{2} / 4 \pi \rho_{0} c^{2} \gg 1 ; \sigma_{0}=30\right.$ was used here) and at rest, whose back leans against a conducting "wall" while its front faces vacuum (from Granot et al. 2011), versus the time $t$ in units of the shell's initial fast magnetosonic crossing time $t_{0} \approx l_{0} / c$. The analytic expectations (dotted and dasheddotted lines) and the results of numerical simulations (diamond symbols joined by a solid line) are in very good agreement. Right: evolution of the typical (or energy-weighted average) Lorentz factor $\Gamma$ with the distance $R \approx c t$ from the central source, for a finite shell similar to that described in the left panel, but for a spherical shell propagating into an external medium with a power-law density profile, $\rho_{\mathrm{ext}}=A R^{-k}$ (from Granot 2012a).

The left panel of Figure 1 shows the results for our impulsive magnetic acceleration test case: a finite cold shell of plasma initially uniform (with width $l_{0}$, rest mass density $\rho_{0}$ and magnetic field $\left.B_{0}\right)$, highly magnetized $\left(\sigma_{0}=B_{0}^{2} / 4 \pi \rho_{0} c^{2} \gg 1\right)$ and at rest, whose back leans against a conducting "wall" while its front faces vacuum. A strong, self-similar rarefaction wave forms at the front of the shell (vacuum interface) and propagates towards its back, reaching the wall at $t=t_{0} \approx l_{0} / c$. By this time the shell's energy-weighted mean Lorentz factor and magnetization are $\langle\Gamma\rangle \sim \sigma_{0}^{1 / 3}$ and $\langle\sigma\rangle \sim \sigma_{0}^{2 / 3}$. At $t>t_{0}$ the shell detaches from the wall, keeps an almost constant width $\left(l \approx 2 l_{0}\right)$ and accelerates as $\langle\Gamma\rangle \sim \sigma_{0} /\langle\sigma\rangle \sim\left(\sigma_{0} t / t_{0}\right)^{1 / 3}$ up to the coasting time $t_{c}=\sigma_{0}^{2} t_{0}$. At $t>t_{c}$ the shell starts coasting at $\langle\Gamma\rangle \sim \sigma_{0}$ while its width grows $\left(l / 2 l_{0} \sim t / t_{c}\right)$ as its magnetization rapidly decreases $\left(\langle\sigma\rangle \sim t_{0} / t\right)$, 
resulting in complete conversion of magnetic to kinetic energy and allowing strong shocks to develop in the flow, which can lead to a large radiative efficiency.

The right panel of Figure 1 shows the evolution of a similar shell in spherical geometry that propagates into an external medium with a power-law density profile, $\rho_{\text {ext }}=A R^{-k}$. The initial shell magnetization $\sigma_{0}$ and density $\rho_{0} \propto 1 / \sigma_{0}$ are allowed to vary while keeping fixed the the values of the initial time or length scale $\left(t_{0} \approx R_{0} / c\right.$ or $\left.R_{0}\right)$, energy $\left(E \sim L t_{0} \approx L R_{0} / c\right.$ or power $\left.L\right)$, and external density $\left(k<2\right.$ in this figure, and $A$ or $\left.\rho_{\text {ext }}\left(R_{0}\right)=A R_{0}^{-k}\right)$, which imply fixed $\Gamma_{\mathrm{cr}} \sim\left(f_{0} \sigma_{0}\right)^{1 /(8-2 k)}$ where $f_{0}=\rho_{0} / \rho_{\text {ext }}\left(R_{0}\right)$ and $R_{\mathrm{cr}} \sim R_{0} \Gamma_{\mathrm{cr}}^{2}$. Shown are the two dynamical regimes most relevant for GRBs. The purple line shows regime I $\left(1<\sigma_{0}<\Gamma_{\text {cr }}\right.$ or a sufficiently low external density) where the shell initially expands as if into vacuum (as described in the left panel) and only after becoming kinetically dominated and expanding radially is it significantly decelerated by the external medium through a strong relativistic reverse shock, that can produce a bright emission that peaks on a timescale larger than the duration of the prompt GRB emission (the familiar low- $\sigma$ "thin shell"; Sari \& Piran 1995). Eventually, most of the energy is transfered to the shocked external medium and the flow approaches the Blandford-McKee (1976) self-similar solution.

The green line shows regime II $\left(1<\Gamma_{\mathrm{cr}}<\sigma_{0}<\Gamma_{\mathrm{cr}}^{3(4-k) / 2}\right)$ where the shell is significantly affected by the external medium while it is still Poynting dominated (at $R>R_{u} \sim R_{0}\left(f_{0} \sigma_{0}^{-1 / 3}\right)^{3 /(10-3 k)}$ ), thus suppressing the reverse shock (which is either non-existent or very weak). The shell remains highly magnetized and gradually transfers its energy to the shocked external medium through $p d V$ work across the contact discontinuity up to $R_{\mathrm{cr}}$, after which the flow approaches the Blandford-McKee solution. In this regime no significant reverse shock emission is expected, and the onset of the afterglow (i.e. the peak of the emission from the shocked external medium) is expected to be on a timescale comparable to the prompt GRB duration (i.e. a high- $\sigma$ "thick shell"). In addition, there are other regimes not shown in this figure. In regime III $\left(1<\Gamma_{\mathrm{cr}}^{3(4-k) / 2}<\sigma_{0}\right)$ the external density is high enough that there is no impulsive acceleration stage where $\langle\Gamma\rangle \propto R^{1 / 3}$, and instead $\langle\Gamma\rangle \sim \sigma_{0} /\langle\sigma\rangle \propto R^{(k-2) / 4}$ at $R_{0}<R<R_{\mathrm{cr}} \sim R_{\mathrm{dec}}$, and then approaches the Blandford-McKee solution (its observational signatures are expected to be similar to regime II). In regime IV $\left(\Gamma_{\mathrm{cr}}<1\right)$ the external density is so high that the flow remains Newtonian all along (as might happen while the GRB jet is propagating inside a massive star progenitor). There is also an exotic regime II* that exists only in a highly stratified external medium $(10 / 3<k<4)$.

In practice, GRB variability times are typically large enough that the flow should first undergo quasi-steady collimation-induced acceleration that saturates, and only later the impulsive acceleration kicks in and operates until the flow becomes kinetically dominated. The effects of multiple sub-shells in the outflow can be important, and the collisions between them may provide efficient energy dissipation that can power the GRB emission (Granot 2012b; Komissarov 2012). They may also allow a low- $\sigma$ "thick shell", i.e. a strong relativistic reverse shock peaking on a timescale comparable to the prompt GRB emission, which is not possible for 
a single shell. For a long-lived source (e.g. AGN) with initial sub-shell widths $l_{0}$ and separations $l_{\text {gap }}$ each sub-shell can expand by a factor of $1+l_{\text {gap }} / l_{0}$, and its magnetic energy decreases by the same factor (where $\sigma_{\infty} \sim l_{0} / l_{\text {gap }}$ ), and may be converted to kinetic or internal energy, or radiation. For a finite source activity, the merged shell can still expand further and convert more magnetic energy into other forms (even without interaction with an eternal medium). Important related points that warrant further study are the transition from impulsive to quasi-steady collimation induced acceleration, both in a single shell and in multiple sub-shells, as well as the dissipation in the interaction between sub-shells and its effect on the outflow acceleration and the resulting emission, such as a possible photospheric spectral component.

\section{Jet dynamics during the afterglow stage}

\subsection{Reconciling between analytic models and numerical simulations}

Here I summarize the main results of Granot \& Piran (2012). Similar to most studies of GRB jet dynamics during the afterglow phase, we focused on an initially uniform jet with well defined, sharp edges. The jet dynamics have been studied mainly analytically (e.g. Rhoads 1999; Sari et al. 1999; Panaitescu \& Kumar 2001) and numerically using two dimensional special relativistic hydrodynamic simulations (e.g. Granot et al. 2001; Zhang \& MacFadyen 2009), as well as with an intermediate "thin shell" approach (Kumar \& Granot 2003) where the dynamical equations are integrated over the radial profile of the shocked fluid (thus reducing them to a set of $1 \mathrm{D}$ partial differential equations). Analytic models predict a rapid sideways expansion, with an exponential growth of the jet half-opening angle $\theta_{\mathrm{j}}$ with radius $R$ at $R>R_{\mathrm{j}}$ where $\Gamma$ drops below $1 / \theta_{0}$. Numerical simulations, however, show a much more modest lateral expansion, with a quasi-logarithmic growth of $\theta_{\mathrm{j}}\left(R>R_{\mathrm{j}}\right)$, where most of the energy remains within the initial jet halfopening angle $\theta_{0}$ until the flow becomes mildly relativistic, and only then does the flow start to gradually approach spherical symmetry. Such a behavior is obtained in analytic models under the crude approximation that the jet does not expand sideways significantly until it becomes non-relativistic (Granot et al. 2005).

Most simulations so far were for $\theta_{0}=0.2$, or even larger $\theta_{0}$. Recently, however, Wygoda et al. (2011) and later van Eerten \& MacFadyen (2012) have performed simulations also for narrower initial jets, $\theta_{0}=0.05,0.1,0.2$. Wygoda et al. (2011) have found that significant lateral spreading starts when $\Gamma$ drops below $\theta_{0}^{-1}$, as predicted by analytic models, and tried to reconcile the apparent discrepancy with analytic models by attributing it to their small range of validity after significant lateral spreading starts $\left(1 \ll \Gamma<\theta_{0}^{-1}\right)$ for the typical modest values of $\theta_{0}$ used in the simulations. van Eerten \& MacFadyen (2012) disagreed with this conclusion.

Granot \& Piran (2012) have reconciled this debate by constructing generalized analytic models that remain valid when the jet becomes wide or sub-relativistic. 
In particular, two different recipes were considered for the lateral expansion,

$$
\frac{d \theta_{j}}{d \ln R}=\frac{\beta_{\theta}}{\beta_{r}} \approx \frac{1}{\Gamma^{1+a} \theta_{j}^{a}}, \quad a= \begin{cases}1 & (\hat{\beta}=\hat{n}), \\ 0 & \left(u_{\theta}^{\prime} \sim 1\right) .\end{cases}
$$

The first, old recipe ( $a=0$, which was used in most previous analytic works), corresponds to a mildly relativistic lateral expansion speed in the jet's comoving rest frame $\left(u_{\theta}^{\prime} \sim 1\right)$. The second, new recipe $(a=1)$, is based on the jump conditions for oblique shocks of arbitrary proper velocity $(u=\Gamma \beta)$, which imply that the velocity of fluid just behind the shock front (in the downstream region) is in the direction of the local shock normal (i.e. perpendicular to the shock front at that location, $\hat{\beta}=\hat{n}$; Kumar \& Granot 2003) in the upstream rest frame.

In addition, two different recipes were considered for sweeping-up the external medium, named after the shape of the swept-up region. In the "trumpet" model external medium is swept-up only at the front of the jet (part of a sphere within a double-sided cone), while in the "conical" model it is also swept-up along its sides, so that once the jet becomes spherical the swept-up mass equals that originally within a sphere of the same radius (while it is smaller in the trumpet model). These two recipes are the basis of two new analytic models, which remain valid for slow, wide jets. For comparison, results are also shown for the old "relativistic" model, which break down when the jet becomes mildly relativistic or wide (and sweeps-up mass similarly to the trumpet model).

The new analytic models fit the results of numerical simulations much better (see left panel of Fig. 2), mainly because they remain valid also in the mildly relativistic, quasi-spherical regime. They show that for modest initial jet half-opening angles, $\theta_{0}$, the outflow is not sufficiently ultra-relativistic when its Lorentz factor reaches $\Gamma=1 / \theta_{0}$ and therefore the sideways expansion is rather slow, showing no rapid, exponential phase. On the other hand, jets with an extremely narrow initial half-opening angle $\left(\theta_{0} \ll 10^{-1.5}\right.$ for $k=0$ or $\theta_{0} \ll 10^{-2}$ for $k=2$; see left panel of Fig. 2), which are still sufficiently ultra-relativistic at $\Gamma=1 / \theta_{0}$, do show a phase of rapid, exponential lateral expansion. However, even such jets that expand sideways exponentially are still not spherical when they become sub-relativistic.

\subsection{An afterglow jet propagating into a stratified medium}

The clear association of long-soft GRBs with Type Ic supernovae, and thus with the death of a massive star, implies that the afterglow shock propagates into the pre-explosion stellar wind. This suggests a stratified external medium with a density profile $\rho_{\text {ext }}=A R^{-k}$. For a constant wind velocity $v_{w}$ to mass-loss rate $\dot{M}_{w}$ ratio, $k=2$ and $A=\dot{M}_{w} /\left(4 \pi v_{w}\right)$. However, as $\dot{M}_{w} / v_{w}$ might vary before the explosion and is rather uncertain, it is worth to also consider other values of $k$.

Here I summarize the results of new 2D special relativistic hydrodynamic simulations by De Colle et al. (2012b), which are the first published simulation results for $k>0$. We performed simulations of the GRB jet dynamics using the Mezcal code (De Colle et al. 2012a) and calculated the resulting afterglow emission, for 

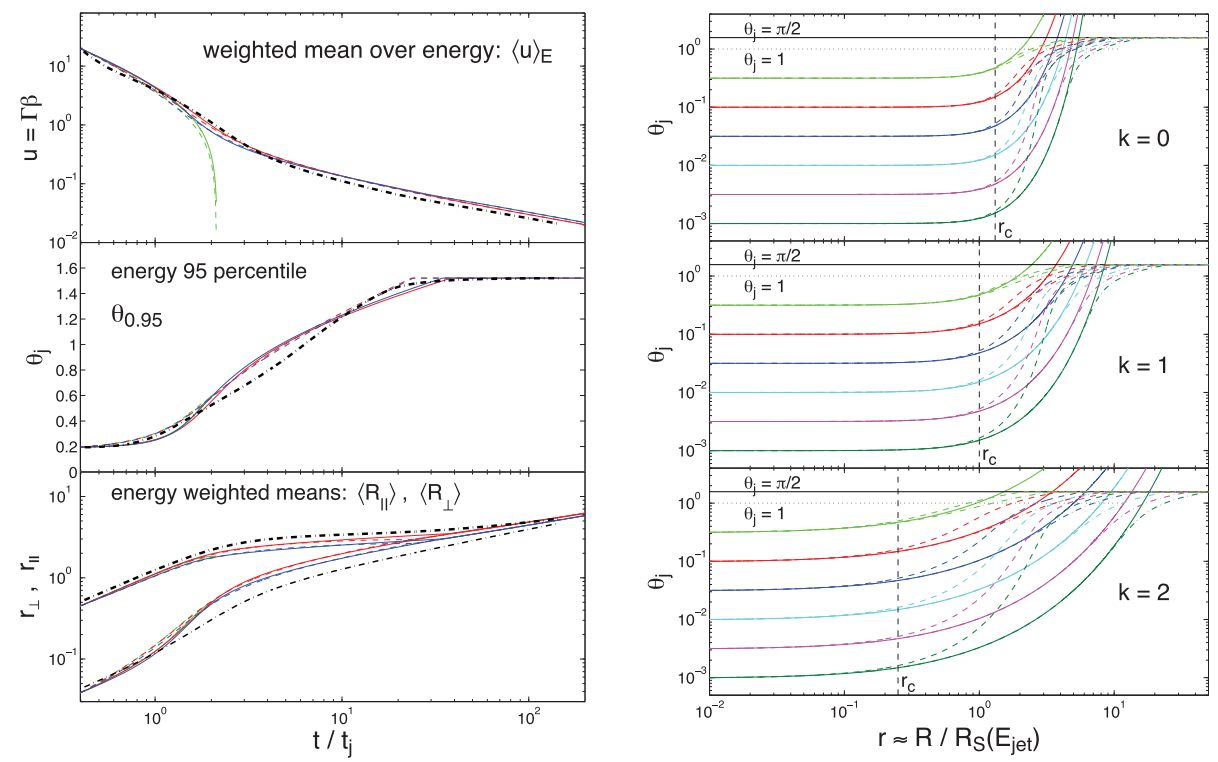

Fig. 2. Left: comparison, for $\theta_{0}=0.2$ and $k=0$ (for an external density profile $\rho_{\text {ext }} \propto$ $R^{-k}$ ), between the analytic models of Granot \& Piran (2012) (thin lines) and the results of 2D special relativistic hydrodynamic simulations (from De Colle et al. 2012a,b) of a jet with initial conditions of a conical wedge of half-opening angle $\theta_{0}$ taken out of the Blandford \& McKee (1976) self-similar solution (thick dot-dashed black line), in terms of the jet proper velocity $(u=\Gamma \beta)$, half-opening angle $\left(\theta_{j}\right)$ as well as normalized parallel $\left(r_{\|}\right)$and perpendicular $\left(r_{\perp}\right)$ sizes. The green, red and blue lines are for the relativistic, trumpet, and conical models, respectively. Thin solid lines are for the new recipe for lateral expansion $(a=1)$ while thin dashed lines are for the old recipe $(a=0)$. Right: comparison between the relativistic (solid lines), trumpet (dot-dashed lines) and conical (dashed lines) models of GP12 in terms of the evolution of the jet half-opening angle $\theta_{j}$ with the normalized radius $r$, for $k=0,1,2$ (top to bottom panels), where all models use our new recipe for the lateral spreading of the jet $(a=1)$. Results are shown for $\log _{10}\left(\theta_{0}\right)=-3,-2.5, \ldots,-0.5$ (using different colors) while the values of $\theta_{0}=1, \pi / 2$ and the critical radius $r_{c}=[(3-k) / 2]^{(3-a) /[(1+a)(3-k)]}$ where the lateral spreading is expected to become significant are shown for reference.

$k=0,1,2$. The initial conditions were taken to be a conical wedge of half-opening angle $\theta_{0}=0.2$ taken from the spherical, self-similar Blandford-McKee solution.

The jet dynamics in stratified external media $(k=1,2)$ are found to be broadly similar to those in a uniform external medium $(k=0)$, and the jet half-opening angle starts increasing logarithmically with time (or radius) once the Lorentz factor $\Gamma$ drops below $\theta_{0}^{-1}$ (as $\theta_{0}$ is modest; see Sect. 3.1). For larger $k$ values, however, the lateral expansion speed is initially faster while $\Gamma>\theta_{0}^{-1}$ but slower at late times, since it increases as $\Gamma$ decreases (e.g., Eq. (3.1)), which in turn occurs more 

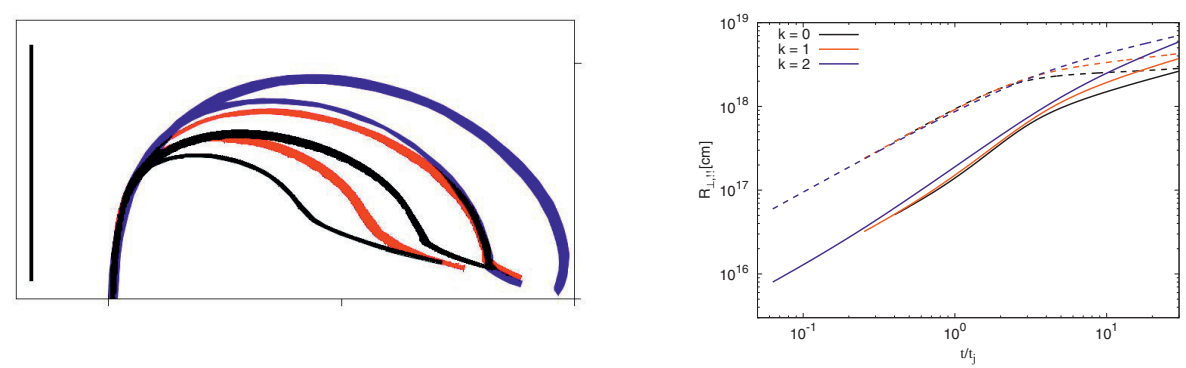

Fig. 3. Left: a comparison between the simulated bow shock structures for $k=0$ (black), $k=1$ (red) and $k=2$ (blue), where $\rho_{\text {ext }} \propto R^{-k}$, at two times that have been selected so that the jet has the same Lorentz factor of 10 and 5 in all simulations. The evolutionary scale unit of $\frac{1}{2} c t$ is indicated with a black transverse bar (the simulations are normalized with respect to $c t$ ). The origin of the axis is located at the right bottom corner and the jet's main direction of propagation is to the left. Right: the transverse $\left(R_{\perp}\right)$ and parallel $\left(R_{\|}\right)$size of the jet, averaged over the total energy excluding rest mass, as a function of the lab frame time in units of the jet break time (from De Colle et al. 2012a).

slowly for larger $k$ (e.g., in the spherical case $\left.\Gamma \propto M^{-1 / 2} \propto R^{(k-3) / 2}\right)$, while $\Gamma\left(t_{\mathrm{j}}\right) \approx \theta_{0}^{-1}$ for all $k$ (such a behavior is also seen in analytic models, e.g., Granot 2007; Granot \& Piran 2012). Therefore, for larger $k$ values the jet is initially wider at the same value of $\Gamma \geq \theta_{0}^{-1}$ (see left panel of Fig. 3), while later on at $\Gamma<\theta_{0}^{-1}$ it becomes Newtonian and approaches spherical symmetry more slowly as its parallel size $R_{\|}$keeps growing (while $R_{\|}$essentially stalls for $k=0$ as the jet becomes sub-relativistic, until the flow approaches spherical symmetry; see right panel of Fig. 3; such a behavior also occurs in analytic or semi-analytic models: Granot et al. 2005; Granot \& Piran 2012; see lower panel of Fig. 2).

The left panel of Figure 4 shows the shape of the jet break for $k=0,1,2$. We find that contrary to analytic expectations (Kumar \& Panaitescu 2000), there is a reasonably sharp jet break in the lightcurve for $k=2$. Moreover, the shape of the jet break is affected more by the viewing angle $\theta_{\text {obs }}$ (within the initial jet aperture, $\left.\theta_{\text {obs }} \leq \theta_{0}\right)$ than by the external density profile slope $k$ (for $0 \leq k \leq 2$ ). Steeper density profiles (i.e. larger $k$ values) are found to produce more gradual jet breaks while larger $\theta_{\text {obs }}$ cause smoother and later appearing jet breaks. For $\theta_{\text {obs }}=0$ most of the steepening occurs within a factor of $\sim 2-4$ in time for $0 \leq k \leq 2$ while for $\theta_{\text {obs }} \sim(0.5-1) \theta_{0}$ it takes $\sim 1-2$ decades for $0 \leq k \leq 2$.

The right panel of Fig. 4 shows the radio lightcurves for the $2 \mathrm{D}$ simulations (black lines). The counter-jet becomes visible as it turns sub-relativistic, and for $k=0$ this results in a clear bump-like feature in the light curve. However, for larger $k$ values the jet decelerates and comes into view more gradually, causing only a mild flattening in the radio light curve that might be hard to discern when $k=2$. This might, however, not help explain the lack of a clear flattening or rebrightening in the late radio afterglow of GRB 030329 (e.g., Pihlström et al. 2007), since in 

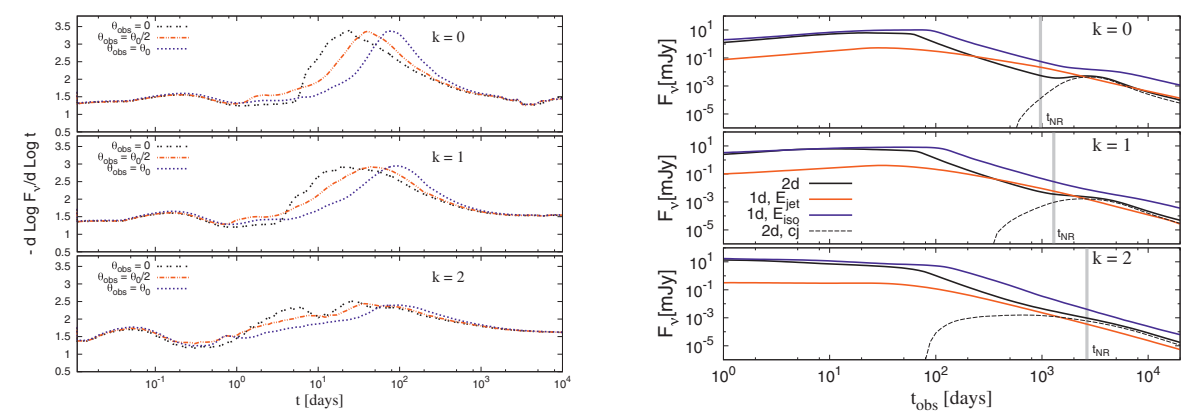

Fig. 4. Left: "jet break shape" - the temporal decay index $\alpha \equiv-d \log F_{\nu} / d \log t_{\mathrm{obs}}$ as a function of the observed time $t_{\text {obs }}$ (including electron cooling, at $\nu=10^{17} \mathrm{~Hz}>\nu_{m}$ ). Right: radio light curves (at $\nu=1 \mathrm{GHz}$ ) for $k=0,1,2$ simulations in 2 d, 1 d with $E=$ $E_{\text {jet }}$, and for a cone with half-opening angle $\theta_{0}$ computed from spherical $1 \mathrm{~d}$ simulations with $E=E_{\text {iso. }}$. The contribution due to the counter-jet is included in the lightcurves, and explicitly shown (dashed curves) for the $2 \mathrm{~d}$ simulations (from De Colle et al. 2012a).

that case detailed afterglow modeling (of the evolution of both the broad band flux densities and the afterglow image size) favors a uniform external density.

The right panel of Figure 4 also shows lightcurves for a spherical 1D simulation with the same true energy (red lines), and for a double-sided cone of half-opening angle $\theta_{0}$ taken from a spherical 1D simulation (from De Colle et al. 2012a) with the same isotropic equivalent energy (blue lines). Late time radio calorimetry usually assumes a spherical flow near the non-relativistic transition time $t_{\mathrm{NR}}$ (thick vertical gray lines), and is thus likely to consistently over-estimate the true energy by up to a factor of a few for $k=2$, but either over-predict or under-predict it by a smaller factor for $k=0,1$ (as can be seen by comparing the red and black lines in the figure).

\section{Conclusions}

Some recent progress in our understanding of GRB jet dynamics has been outlined. A strongly variable initially Poynting flux dominated outflow can convert most of the initial magnetic energy into kinetic and internal energy, thus allowing high radiative efficiencies from dissipation within the outflow - internal shock at a range of magnetizations. More generally, as our understanding of initially highly magnetized outflows improves, they are gradually becoming a more viable alternative to the traditional fireball model, making them worthy of further study.

The apparent discrepancy between the results of analytic models and numerical simulation for the degree of jet lateral expansion during the afterglow was finally reconciled. An early phase of exponential sideways expansion with radius occurs only for extremely narrow jets $\left(\theta_{0} \ll 0.05\right.$ for $k=0$ or $\theta_{0} \ll 0.01$ for $k=2$ ) but is replaced by a slower, quasi-logarithmic sideways expansion for more modest 
initial half-opening angles $\left(\theta_{0} \gtrsim 0.05\right.$ for $k=0$ or $\theta_{0} \gtrsim 0.01$ for $\left.k=2\right)$. The jet first becomes sub-relativistic, and only then gradually approaches spherical symmetry.

Afterglow jets propagating into a stratified external medium $(k>0)$ expand sideways faster before the jet break and slower afterwards, compared to a uniform external medium $(k=0)$. Thus, they become spherical and sub-relativistic more slowly, which makes it hard to see a signature of their counter-jet in the lightcurve. Their jet break is generally smoother, but potentially detectable, and its sharpness depends more on the viewing angle (for $\theta_{\text {obs }}<\theta_{0}$ ) than on $k$ (for $0 \leq k \leq 2$ ).

\section{References}

Abdo, A.A., Ackermann, M., Arimoto, M., et al., 2009, Science, 323, 1688

Begelman, M.C., \& Li, Z.-Y. 1992, ApJ, 397, 187

Blandford, R.D., 2002, in Lighthouses of the Universe, ed. M. Gilfanov, et al. (SpringerVerlag, Berlin), 381

Blandford, R.D., \& McKee, C.F., 1976, Phys. Fluids, 19, 1130

Blandford, R.D., \& Rees, M.J., 1974, MNRAS, 169, 395

Contopoulos, J., 1995, ApJ, 450, 616

De Colle, F., Granot, J., Lopez-Camara, D., et al., 2012a, ApJ, 746, 122

De Colle, F., Ramirez-Ruiz, E., Granot, J., et al., 2012b, ApJ, 751, 57

Drenkhahn, G., \& Spruit, H.C., 2002, A\&A, 391, 1141

Goldreich, P., \& Julian, W.H., 1970, ApJ, 160, 971

Granot, J., 2005, ApJ, 631, 1022

Granot, J., 2007, Rev. Mex. A\&A, 27, 140

Granot, J., 2012a, MNRAS, 421, 2442

Granot, J., 2012b, MNRAS, 421, 2467

Granot, J., Komissarov, S.S., \& Spitkovsky, A., 2011, MNRAS, 411, 1323

Granot, J., Miller, M., Piran, T., et al., 2001, in "GRBs in the Afterglow Era", ed. E. Costa, F. Frontera \& J. Hjorth (Berlin, Springer), 312

Granot, J., \& Piran, T., 2012, MNRAS, 421, 570

Granot, J., Ramirez-Ruiz, E., \& Loeb, A., 2005, ApJ, 618, 413

Heinz, S., \& Begelman, M.C., 2000, ApJ, 535, 104

Komissarov, S.S., 2012, MNRAS, 422, 326

Komissarov, S.S., Vlahakis, N., Königl, A., et al., 2009, MNRAS, 394, 1182

Komissarov, S.S., Vlahakis, N., \& Königl, A., 2010, MNRAS, 407, 17

Kumar, P., \& Granot, J., 2003, ApJ, 591, 1075

Kumar, P., \& Panaitescu, A., 2000, ApJ, 541, L9

Levinson, A., 2010, ApJ, 720, 1490

Lyubarsky, Y.E., 2009, ApJ, 698, 1570

Lyubarsky, Y.E., 2010, MNRAS, 402, 353

Lyubarsky, Y.E., 2010, ApJ, 725, L234

Lyutikov, M., 2006, New J. Phys., 8, 119

Lyutikov, M., 2011, MNRAS, 411, 422 
Panaitescu, A., \& Kumar, P., 2001, ApJ, 554, 667

Pihlström, Y.M., Taylor, G.B., Granot, J., et al., 2007, ApJ, 664, 411

Rhoads, J.E., 1997, ApJ, 487, L1

Rhoads, J.E., 1999, ApJ, 525, 737

Sari, R., Piran, T., \& Halpern, J., 1999, ApJ, 519, L17

Sari, R., \& Piran, T., 1995, ApJ, 455, L143

Smith, M.D., Norman, M.L., Wilson, J.R., et al., 1981, Nature, 293, 277

Tchekhovskoy, A., Narayan, R., \& McKinney, J.C., 2010, New Astron., 15, 749

van Eerten, H.J., \& MacFadyen, A.I., 2012, ApJ, 751, 155

Vlahakis, N., \& Königl A., 2003, ApJ, 596, 1080

Wygoda, N., Waxman, E., \& Frail, D.A., 2011, ApJ, 738, L23

Zhang, W., \& MacFadyen, A.I., 2009, ApJ, 698, 1261 